\title{
SEELE und SCHMERZ
}

\section{André Thurneysen}

Dr. med., Facharzt für Allgemeinmedizin, SIM-Gutachter, em. Dozent für Homöopathie Medizinische Fakultät Bern, Mitglied FMH

\section{Einleitung}

In den letzten Wochen haben 5 Artikel in der SÄZ mich zu den nachstehenden Überlegungen veranlasst, insbesondere die Frage nach einem neuen Menschenbild sowie die dialektische Auseinandersetzung zwischen Schmerz und Leiden.

- Piet van Spijk. Die Medizin: Auf der Suche nach einem neuen Menschenbild [1].

- Roland Schreiber. Chronische Schmerzen und Arbeitsfähgkeit [2].

- Walter Kissel. Das somatoforme Schmerzsyndrom [3].

- Rolf H. Adler. Nicht jedes Leiden braucht eine Diagnose [4].

- Hedi Meierhans. Quantenphysik - Basis für ein neues Weltbild (Leserbrief) [5].

\section{Schmerzen - Leiden}

Bevor wir diese dialektische Auseinandersetzung vertiefen können, müssen wir insbesondere den Begriff der Gesundheit klären. Man kann diese, wie dies in den letzten Jahren allgemein anerkannt wurde, als biopsycho-soziales Wohlbefinden definieren. Diese Definition eines Zustandes greift meines Erachtens etwas kurz und ich ziehe es vor, Gesundheit als die Fähigkeit eines lebenden Organismus, sich in einem durchaus bio-psycho-sozialen Gleichgewicht halten zu können, $\mathrm{zu}$ beschreiben. Dies entspricht einer dynamischen,

\section{Ich ziehe es vor, Gesundheit als die Fähigkeit eines lebenden Organismus, sich in einem durchaus bio-psycho-sozialen Gleichgewicht halten zu können, zu beschreiben.}

ständig aktiv zu erbringenden Leistung angesichts aller Herausforderungen des täglichen Lebens (wie z.B. Klima, Ernährung, Infekte, Verletzungen, Aggressionen, Sorgen, Demütigungen, Entwurzelung, u. a.m.). Kann dieses dynamische Gleichgewicht nicht aufrechterhalten werden (dank den uns immer noch unbekannten sogenannten Selbstheilungskräften), meldet uns der Organismus kraft der ihm innewohnenden Lebenskraft mittels Symptomen, dass etwas (noch) nicht stimmt. Der Schmerz ist ein solches Symptom, welches in seiner akuten Manifestation mehr oder weniger lokalisierbar ist und oft einer gestörten körperlich-leiblichen Struktur zogeordnet werden kann. Dies führt in der Regel auch zu einer Diagnose, welche, wie wir dies alle gelernt haben, vor dem therapeutischen Entscheid zu stehen hat. Im Falle einer physischen Verletzung, Fraktur, Kolik oder Entzündung ist die Evidenz meist unbestritten und die sinnvolle Therapie kann durchgeführt werden.

\section{Der Schmerz bekommt eine neue Bedeutung, er wird zum Leiden.}

Nun wissen wir ebenfalls alle, wie dies die Kollegen Schreiber [2], Kissel [3] und Adler [4] beschrieben und diskutiert haben, dass sich Schmerz chronifizieren kann, dass er den Bezug zur initialen Läsion aufgeben und sich verselbständigen kann, wie die neueren neurophysiologischen Forschungen detailliert aufgezeigt haben. Der Schmerz bekommt eine neue Bedeutung, er wird zum Leiden. Perfiderweise kann er aber durchaus eine Lokalisation am/um den Ort eines ehemals akuten, mittlerweile abgeheilten Geschehens beibehalten, was, zumindest für die betroffene Person, den Zusammenhang mit einer tieferen, nicht materiellen Ursache seines Leidens nicht oder nur schwierig nachvollziehbar macht.

Die von Kollege Kissel [3] beschriebene Differenzierung zwischen primärer (meist depressiver) und sekundärer (meist muskulärer) Genese dieser chronischen Schmerzzustände ist sehr wichtig, obschon meiner Erfahrung als SIM-Experte nach die Überschneidungen und Mischformen sehr häufig und komplex sind. Dies macht auch die Begutachtungen solcher Situationen so schwierig, was sowohl Kollege Schreiber [2] als auch Kollege Kissel [3] klar formuliert haben.

Wir sind angesichts dieser Patienten mit einem Leiden konfrontiert, welches einmalig individuell empfunden und exprimiert wird und sich eben nicht in eine Diagnose (ICD-10 F45.4), einen Raster, eine messbare Grösse oder eine Prozentzahl pressen lässt [4]. Die Erfassung eines solchen Leidens, einer solchen Situation war schon immer, ist und bleibt eine ärztliche Tätigkeit und Pflicht. Es geht darum, die leidende Person in ihrer Gesamtheit mit all ihren Facetten, Sorgen, Konflikten, Demütigungen einerseits, ihrer körperlichen Leiblichkeit [3] andrerseits zu erfassen, zu verstehen, zu erfühlen und ihr damit auch den hippokratischen Respekt zu zollen und ihre Würde zu bewahren. 
Die bio-psycho-soziale Methode, die Homöopathie, die TCM, die ayurvedische Medizin haben solche Annäherungen entwickelt und formuliert.

\section{Es geht darum, die leidende Person in ihrer Gesamtheit mit all ihren Facetten zu erfassen.}

Dazu braucht es kein neues Menschenbild, sondern einen gesunden Menschenverstand, Empathie und einen erweiterten Menschenblick.

Das Bundesgerichtsurteil von Juni 2015 ist der Versuch der Juristen, einen Schritt in diese Richtung zu gehen. Ich wünschte mir, dass die IV im Sinne einer Anerkennung dieser Leiden mit dem Zusprechen einer (evtl. zeitlich begrenzten) Teilrente diesen Patienten den erwähnten Respekt eines leidenden, eben auch in seiner Arbeitsfähigkeit reduzierten Individuums zollen könnte, was Platz liesse für die Würde des betroffenen Menschen sowie die Hoffnung auf eine weiterhin aktive, sinnvolle Existenz. Dies vermöchte die von Kollege Schreiber [2] erwähnte Motivationshürde zu senken und könnte auch einen positiven Einfluss auf die therapeutische Rezeptivität haben. Es geht schliesslich darum, den fatalen Teufelskreis zu durchbrechen oder, wenn früh genug evaluiert, gar nicht erst entstehen zu lassen.

\section{Ausblick}

Ich möchte auf die Frage des Menschenbildes und unserer Seele zurückkommen.

Wir wissen heute, dass Pflanzen ein Bewusstsein haben, dass Bäume miteinander kommunizieren, dass Tiere zu Wahrnehmungleistungen fähig sind, die wir uns kaum vorstellen können. R. Sheldrake hat seine morphogenetischen Felder postuliert und beschrieben, C. G. Jung hat das Phänomen der Synchronizität untersucht und beschrieben. Es gibt unzählige Berichte über Informationstransfers lebender Organismen, die uns zwingen, andere neue Formen der Informationsübermittlung zu akzeptieren, zu evaluieren und auch zu nutzen.

Erinnern Sie sich an den Augenblick Ihrer ersten Verliebtheit: da waren Sie im Bruchteil einer Sekunde ein völlig anderes Wesen! Da genügen weder $120 \mathrm{~m} / \mathrm{sec}$ Nervenleitgeschwindigkeit noch 20 Minuten Hormonspiegelanpassung. Hier wurde über die Matrix des Bindegewebes (unser körpereigenes «Internet») mit Elektronentransfer (= Lichtgeschwindigkeit) der gesamte Organismus schlagartig informiert und neu konditioniert. Man kann, etwas vereinfacht ausgedrückt, die Intensität unserer Empfindungen (Schönheit, Musik, zwischenmenschliche Gefühle) als eine Form von Reso- nanzphänomen charakterisieren, beruhend auf der Tatsache, dass alles im Universum letztlich Schwingung ist.

Die moderne Quantenphysik [5] stösst an diese Grenzen, weil das Phänomen der Verschränkung («entanglement») der Elementarteilchen über Raum und Zeit hinweg uns mit allem und jedem im Universum verbindet (EPR-Paradoxon).

Es ist die nicht fassbare, nicht messbare, spirituell-geistige Information des einzelnen, einmaligen Individuums, welche das materielle Substrat unserer Existenz belebt und durchdringt und dieses Substrat zur Expression von Symptomen, also zu einer Mitteilung, veranlasst und benutzt als Ausdruck einer gestörten Harmonie (wie eine Art «Software-Hardware»-Funktion) und uns gleichzeitig mit dem gesamten Universum verbindet. $\mathrm{Ob}$ man diese geistige Entität Seele nennen will oder nicht, ist persönliche Ermessenssache, aber diese Entität ist unzerstörbar, unsterblich und bereichert sich mit jeder Erfahrung jeder einzelnen menschlichen, aber auch pflanzlichen oder tierischen Existenz, d.h. diese gewonnene, erfahrene Information bleibt nach unserem Tode erhalten und trägt so zum Erfahrungsschatz des jeweiligen Kollektivs und des Universums bei. Medial veranlagte Wesen haben Zugriff auf diese Information. Wir Menschen sind jeder ein offenes System von offenen Untersys-

\section{Es braucht kein neues Menschenbild.}

temen und gleichzeitig ein individueller, einmaliger Anteil des Ganzen, sodass wir nicht in der Lage sein können, das Funktionieren des Kosmos unvoreingenommen, quasi von aussen, zu betrachten und zu beurteilen. Die Kernphysiker des CERN wissen, dass bereits das Nachdenken über eine Versuchsanordnung diese beeinflusst. Die Energiequelle oder -form, die das Universum steuert oder regelt, entzieht sich unserer Wahrnehmung, weil wir selber darin verschränkt sind [5]. Deshalb braucht es kein neues Menschenbild, sondern einen erweiterten, über das Cartesianische hinausgehenden, post-materialistischen Blick aufs Ganze [6].

Literatur

1 Van Spijk P. Die Medizin: Auf der Suche nach einem neuen Menschenbild. Schweiz Ärzteztg. 2018; 99(19-20): 633-4.

2 Schreiber R. Chronische Schmerzen und Arbeitsfähigkeit. Schweiz Ärzteztg. 2018; 99(22): 724-6.

3 Kissel W. Das somatoforme Schmerzsyndrom. Schweiz Ärzteztg. 2018; 99(23): 768-71.

4 Adler R. Nicht jedes Leiden braucht eine Diagnose. Schweiz Ärzteztg. 2018; 99(24): 814-5.

5 Meierhans H. Quantenphysik - Basis für ein neues Weltbild (Leserbrief). Schweiz Ärzteztg. 2018; 99(24): 797-8.

6 http://opensciences.org/about/manifesto-for-a-post-materialistscience 